\title{
Modified Ipsilateral Endonasal Endoscopic Trans- Sphenoidal Approach to Sphenoid Sinus Lateral Recess Cerebrospinal Fluid Leak Management in Two Cases: A Technical Note
}

\author{
Survendra Kumar R. Rai ${ }^{1}$ Saswat Kumar Dandpat ${ }^{1}$ \\ ${ }^{1}$ Department of Neurosurgery, King Edward Memorial Hospital, \\ Seth Gordhandas Memorial Hospital, Mumbai, Maharashtra, India
}

\begin{abstract}
Address for correspondence Survendra Kumar R. Rai, DNB, Department of Neurosurgery, King Edward Memorial Hospital, Seth Gordhandas Memorial Hospital, Acharya Donde Marg, Parel, Mumbai, Maharashtra, 400012, India (e-mail: rskrrai@gmail.com).
\end{abstract}

Abstract

Keywords
- sphenoid sinus lateral
recess
- transpterygoid
approach
- trans-sphenoidal
approach

Background Cerebrospinal fluid (CSF) leak from the sphenoid sinus lateral recess (SSLR) is very rare. Majority prefer transpterygoid approach which is extensive and time consuming. Two such cases were managed with least possible dissection/destruction of paranasal sinus.

Methods Two cases of SSLR were accessed through the ipsilateral nostril from the side of CSF leak. Wide ipsilateral anterior sphenoidotomy was done preserving intersinus septum of sphenoid sinus. Middle turbinate was lateralized and remaining paranasal structures were preserved. Two handed single nostril approach was done in both the cases by 45 - and 70-degree endoscope along with angled instruments. SSLR defects were visualized and packed with autologous fat graft and glue.

Results SSLR defects could be visualized and packed with fat graft in both the cases from ipsilateral side. Both cases had uneventful outcome with no leak with mean follow-up of 11.5 months.

Conclusion Modified ipsilateral endonasal endoscopy trans-sphenoidal approach is least invasive technique for SSLR leak. Use of angled scope and instruments help in defect visualization, avoiding extensive paranasal sinus dissection and bony removal. Tedious time-consuming reconstructive procedures can be avoided with simple fat graft with good outcome.

\section{Introduction}

Charles Miller described cerebrospinal fluid (CSF) rhinorrhea first in the year 1826. Walter Dandy was the first to perform intracranial repair of CSF leak. Wigand started endoscopic repair which became the standard of care for this entity.

CSF leak due to anterior cranial fossa defect is known entity and its endoscopic repair is well described in literature. However, leak from middle cranial fossa defect is very rare. Transcranial microscopic repair is challenging and fraught with dangers due to risk of injuring neurovascular structures. Endonasal endoscopic approach provides direct access to this area and deals with eloquent structure. SSLR is most common site of CSF leak in middle cranial fossa. Due to lateral skull base location of the defect, its accessibility with simple endoscopic endonasal trans-sphenoidal route is difficult. So, various extended techniques are described in literature.

We have described our experience in managing two cases of spontaneous CSF rhinorrhea due to SSLR defect with a novel technique. 


\section{Case 1}

An 18-year-old male presented with history of watery discharge from right nostril for 2 months. CSF leak was confirmed with $\beta-2$ transferrin test. Computed tomography (CT) scan of sella and paranasal sinus and magnetic resonance (MR) cisternogram were done. Defect was confirmed in right SSLR ( - Fig. 1A, B). He had a history of congenital hydrocephalus for which he underwent right side ventriculo-peritoneal shunt. Patients head size did not improve and so underwent metrizamide CT scan of brain and was diagnosed to have third ventricular arachnoid cyst. Right frontal craniotomy and excision of cyst was done. After 5 years, left-sided ventriculoperitoneal shunt was performed due to shunt malfunction. Recent imaging studies did not show any change in ventricular size. Clinically ventriculoperitoneal shunt was found to be functioning.

\section{Case 2}

A 35-year-old female presented with watery discharge from right nostril for 3 months. No other neurological signs or symptoms were found. CSF leak was confirmed by $\beta-2$ transferrin test. Right SSLR was confirmed by magnetic resonance-cisternogram and CT scan (-Fig. 2).

\section{Procedure}

Both patients were operated with 0-, 45-, or 70-degree rigid scope and with angled instruments. Ipsilateral single-nostril endoscopic approach was performed in both cases. Cottonoid patty soaked with 1:100,000 adrenaline was used to decongest the nasal mucosa. We approached directly through the sphenoid sinus from the ipsilateral side. Middle turbinate was lateralized, superior turbinate was left undisturbed. Sphenoid ostium was enlarged till vidian canal (VC) without exposing the nerve laterally and median sphenoid septum was left undisturbed. Special attention was given to avoid damaging midline sphenoid septum. Mucosa of the sphenoid sinus was removed. Ipsilateral sella and optico-carotid recess was visualized. The defect was seen and once CSF leak was confirmed on Valsalva maneuver. Mucosa around the defect site was removed with angled instruments (-Fig. 2B). Autologous fat graft harvested from the lateral aspect of thigh was used for the sinus packing. Initially, small multiple pieces of fat were kept near the defect site and later larger pieces of fat were placed within the sinus. Median sphenoid septum was used as buttress to tightly pack the sinus with fat. Fibrin glue was also used during the procedure. Nasal mucosa and turbinates were preserved. Exposure of ethmoids were not necessary. Our bony work was up to the medial border of
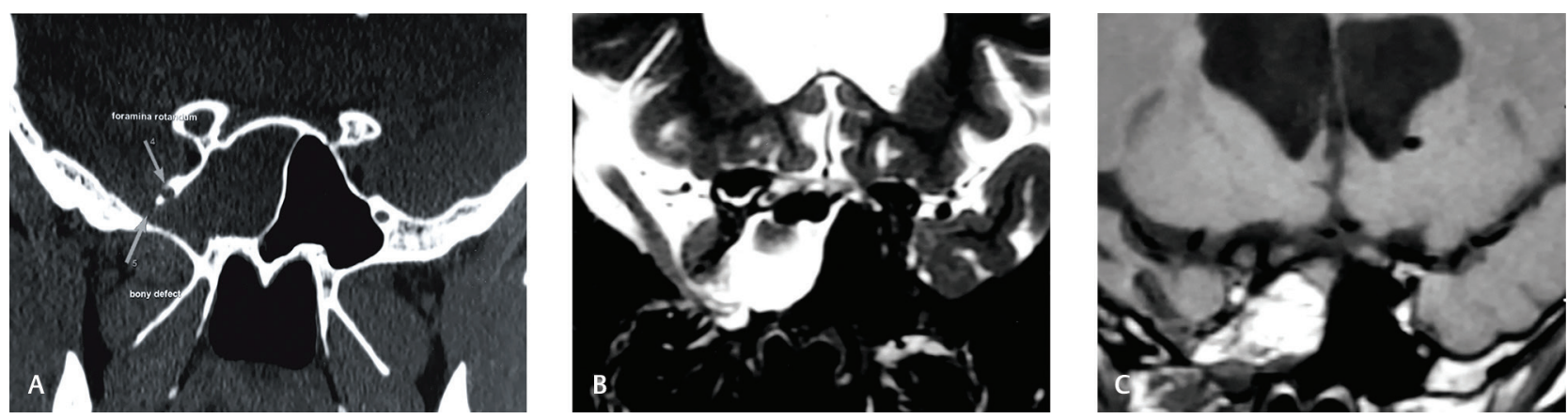

Fig. 1 (A) Thin cut coronal computed tomography image of sella and paranasal sinus showing defect in right SSLR. (B) T2W coronal image of MRI showing cerebrospinal fluid filling the sphenoid sinus through the defect of right SSLR. (C) Postoperative T1W coronal image of MRI showing fat in situ (both intra- and extracranially). MRI, magnetic resonance imaging; SSLR, sphenoid sinus lateral recess.
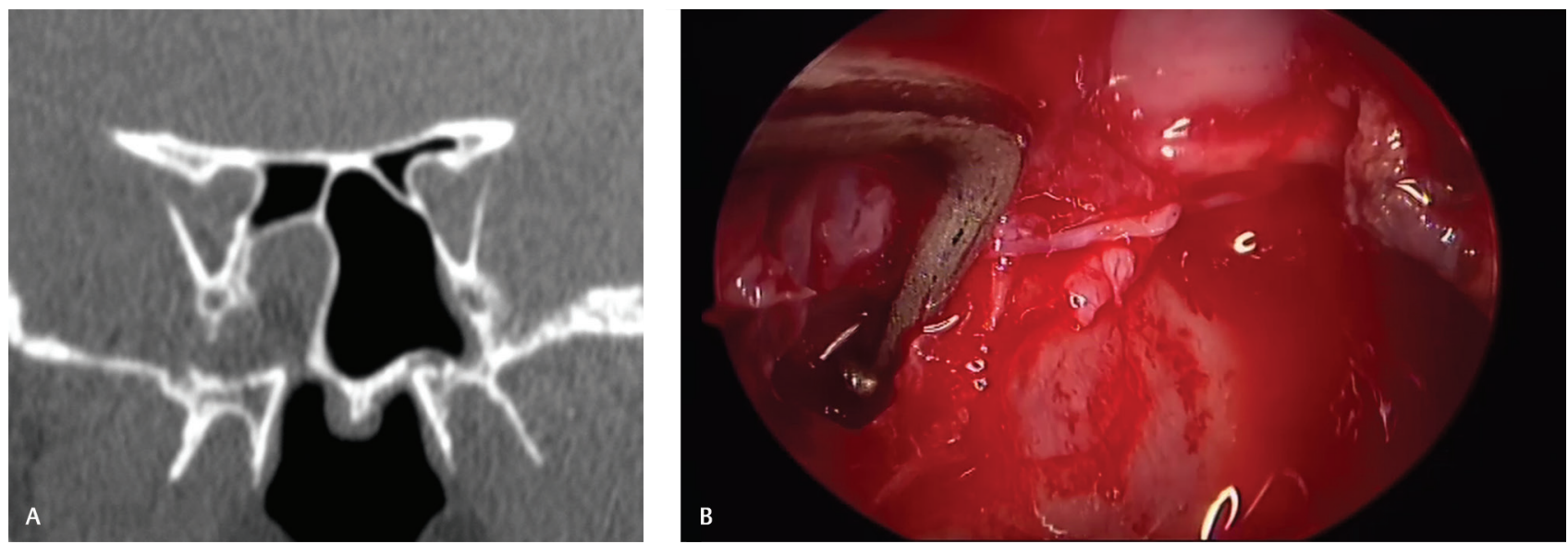

Fig. 2 (A) Thin cut coronal computed tomography image of sella and paranasal sinus showing defect in left SSLR. (B) Intraoperative image showing the defect in the SSLR with angled instrument. SSLR, sphenoid sinus lateral recess. 
VC without exposing the vidian nerve. Visualization of the defect is not perpendicular as in transpterygoid approach. Use of angled endoscope and instruments permit dissection in more medial and superior direction. Modified ipsilateral endonasal endoscopy trans-sphenoidal approach (MIEETSA) is a minimally invasive technique and different from the existing approaches in literature.

Lumbar drainage of CSF was not required postoperatively. Patients were discharged after 5 days of hospital stay. In first case, after 14 months of follow-up, there was no evidence of leak and fat was seen filling the defect and the ipsilateral sphenoid sinus ( - Fig. 1C). In second case, patient was discharged uneventfully. She followed up at 9 months without evidence of any leak. New scan was advised, but she lost to follow up.

\section{Discussion}

The Vidian-Rotundum line ( $\mathrm{V}-\mathrm{R}$ line) connect the medial edges of the anterior opening of the $\mathrm{VC}$ to the extracranial end of the foramen rotundum (FR) and demarcate between the sphenoid body and lateral parts of the sphenoid bone, which include the greater wings and pterygoid process. The lateral recess is defined as the lateral air cells that extends laterally between the VC and the FR and beyond the sphenoid body into the greater wing and/or pterygoid process. Wang et al has classified SSLR into three types based on CT and magnetic resonance imaging: greater wing (12\%), pterygoid $(11 \%)$, or full lateral types (77\%). ${ }^{1,2}$

Spontaneous CSF leak from SSLR though very rare but is the most common location in middle cranial fossa. The cause of it can be ascertained to single cause: benign intracranial hypertension. Sternberg canal has been wrongly posited in literature as an important cause of CSF rhinorrhoea from SSLR. In 2011, Barañano et al studied 1,000 patients radiologically and found leak in only one patient which was consistent with Sternberg canal location. ${ }^{3}$ Sternberg canal is situated medial to FR and superior orbital fissure. Most common site of CSF leak is lateral to FR in pneumatized SSLR. Illing et al suggested that CSF leak due to skull base defect is an acquired pathology. ${ }^{3}$ SSLR CSF leak is due to combination of lateral recess pneumatization, attenuated sphenoid sinus recess roof, and skull base arachnoid pits developing due to intracranial hypertension is another predisposing factor. ${ }^{4}$

Confirmation of diagnosis is done clinically, biochemically, and radiologically. Clinically patients usually present with symptoms of benign intracranial hypertension and uni-nostril or rarely bi-nostril CSF rhinorrhea. Sphenoid leak is likely in cases with gush of watery fluid coming out on forward bending. $\beta-2$ transferrin is the most definitive biochemical test to confirm CSF rhinorrhea. High-resolution thin cut plain CT scan of sella and PNS is required for localizing the bony defect and surgical planning. Difficulty exists in localizing exact site of leak as defect may not be actively leaking or defect may be present without leak. Hence, CT cisternogram can localizes active leak. However, possibility of missing intermittent leak is high with sensitivity ranging from 48 to $96 \%{ }^{5}$ MR cisternography localizes active CSF leak non-invasively and reveals associated encephalocele or meningocele. This helps in localizing intermittent and low CSF leak by fast spin-echo sequence with fat suppression and image reversal to highlight CSF. Magnetic resonance angiography may also help in avoiding complications like aneurysm related dehiscence of sphenoid sinus, cavernous carotid artery in opacified lateral recess. Unfortunately, MRI lacks bony details, which limits its accuracy. The sensitivity has been reported 85 to $92 \%$ with $100 \%$ specificity. ${ }^{5,6}$ Radiolabeled cisternogram and intrathecal fluorescein has low accuracy. ${ }^{6,7}$

Various endoscopic approaches have been described, but all can be put together under three categories: the endoscopic endonasal trans-sphenoid approach (EETSA), the endoscopic endonasal transethmoidal-sphenoidal approach (EETESA), and the endoscopic endonasal trans-pterygoid approach (EETPA). The sphenoid leak can be divided into two types by V-R line: medial and lateral leak. SSLR leak is lateral to the V-R line. Medial leaks are approached though EETSA or EETESA according to the anatomical and radiological location of the defect. As SSLR leaks are lateral and angled in location, it is most commonly approached by transpterygoid route (EETPA). Transpterygoid approach was first described by Bolger in 2006 to repair SSLR leak. ${ }^{8}$ It is currently the most popular technique providing direct access to lateral recess but requires extensive nasal work with drilling of pterygoid process. Extensive dissection adjacent to the neurovascular structure structures make it vulnerable to injury. This increases operative time and requires steep learning curve. Modification of the transpterygoid approach has been tried by few with lesser bony drilling. ${ }^{9}$

Techniques of approaching SSLR by trans-sphenoidal route has been described by very few. Turner et al managed six cases by EETSA or partial transpterygoid approaches to access SSLR CSF leaks. ${ }^{10}$ Stereotactic navigation were used in all cases. In some cases, resection of small part of the sphenoid process of the palatine bone was required for accessing SSLR. He concluded that most of the lateral recess leaks can be managed by transnasal trans-sphenoidal route with angled scope and instruments. No patients had any complications or leak at the end of follow-up period.

Kirtane et al performed uncinectomy, anterior and posterior ethmoidectomy, cutting off posterior end of middle turbinate, widening of sphenoidotomy laterally to reach lateral recess, and Hadad flap for covering the defect. ${ }^{11}$ El-Tarabishi et al used four-handed two nostrils endoscopic trans-sphenoidal approach. ${ }^{9}$ Superior turbinate was resected, middle turbinate sacrificed partially, and rostrum and posterior $1 \mathrm{~cm}$ of nasal septum sacrificed. Bilateral sphenoidotomy was done in all cases and extended laterally to visualize the medial orbital wall and lateral wall of the sphenoid sinus. In his seven patient's series, there was no recurrence of leak after following up for 37 to 48 months.

MIEETSA is different from the existing approaches described in literature. This technique is the most conservative approach with minimal dissection and damage to the nasal mucosa and turbinates. Ethmoids were left undisturbed. Our bony work is up to the medial border of VC without exposure of the nerve. Although view is not perpendicular as 
in transpterygoid approach, but the dissection is possible by suitable angled instrument and use of 45- or 70-degree endoscope. Small pieces of autologous fat graft were kept near the defect site initially while in final stage of packing a single larger piece sufficient to fit snugly within ipsilateral sphenoid sinus was kept. The intact inter-sinus septum of the sphenoid acted like buttress to keep fat graft in position. Except sphenoidotomy no other bony work was done. This prevents new site of CSF leak, neurovascular injury, and faster healing.

\section{Conclusion}

MIEETSA is least invasive technique for SSLR leak. Use of angled scope and instruments help in avoiding extensive paranasal sinus dissection and bony removal. Tedious time-consuming reconstructive procedures can be avoided with simple fat graft with good outcome.

\section{Funding}

None.

\section{Conflict of Interest}

None declared.

\section{Acknowledgments}

The authors would like to thank Dr. Cigdem Kalaycik Ertugay, Associate Professor Department of ENT, Istanbul Training and Research Center, Istanbul, Turkey for her valuable suggestion and help in manuscript preparation.

\section{References}

1 Wang J, Bidari S, Inoue K. Yang H, Rhoton A Jr. Extensions of the sphenoid sinus: a new classification. Neurosurgery 2010;66(4):797-816

2 Ulu MO, Aydin S, Kayhan A, et al. Surgical management of sphenoid sinus lateral recess cerebrospinal fluid leaks: a single neurosurgical center analysis of endoscopic endonasal minimal transpterygoid approach. World Neurosurg 2018;118:e473-e482
3 Illing E, Schlosser RJ, Palmer JN, Curé J, Fox N, Woodworth BA. Spontaneous sphenoid lateral recess cerebrospinal fluid leaks arise from intracranial hypertension, not Sternberg's canal. Int Forum Allergy Rhinol 2014;4(3):246-250

4 Barañano CF, Curé J, Palmer JN, Woodworth BA. Sternberg's canal: fact or fiction? Am J Rhinol Allergy 2009;23(2):167-171

5 Schlosser RJ, Bolger WE. Nasal cerebrospinal fluid leaks: critical review and surgical considerations. Laryngoscope 2004;114(2):255-265

6 Woodworth BA, Bolger WE, Schlosser RJ. Nasal cerebrospinal fluid leaks and encephaloceles. Oper Tech Otolaryngol Neck Surg 2006;17(2):111-116

7 Woodworth BA, Prince A, Chiu AG, et al. Spontaneous CSF leaks: a paradigm for definitive repair and management of intracranial hypertension. Otolaryngol Head Neck Surg 2008;138(6):715-720

8 Bolger WE. Endoscopic transpterygoid approach to the lateral sphenoid recess: surgical approach and clinical experience. Otolaryngol Head Neck Surg 2005;133(1):20-26

9 El-Tarabishi MN, Fawaz SA, Sabri SM, El-Sharnobi MM, Sweed A. A modification of endoscopic endonasal approach for management of encephaloceles in sphenoid sinus lateral recess. Eur Arch Otorhinolaryngol 2016;273(12):4305-4314

10 Turner JH, Dubin M, Quinones-Hinojosa A, Boahene DK. Repair of CSF leaks in the lateral recess of the sphenoid sinus transsphenoid vs. transpterygoid approach. Laryngoscope 2011;121(S4) :S241-S241

11 Kirtane MV, Lall A, Chavan K, Satwalekar D. Endoscopic repair of lateral sphenoid recess cerebrospinal fluid leaks. Indian J Otolaryngol Head Neck Surg 2012;64(2):188-192 